\begin{abstract}
¿Cómo citar este artículo?
Lizarazo López, L. M., Burbano Pantoja, V. M. A. y Valdivieso Miranda, M. A. (mayo-agosto, 2020). Correlación entre actividad física y autoestima de escolares adolescentes: un análisis de tipo trasversal. Revista Virtual Universidad Católica del Norte, (60), 95-115. https://www.doi.org/10.35575/rvucn.n60a6
\end{abstract}

\title{
Correlación entre actividad física y autoestima de escolares adolescentes: un análisis de tipo trasversal*
}

\begin{abstract}
Correlation between physical activity and self-esteem of adolescent schoolchildren: a cross-sectional analysis
\end{abstract}

\section{Laura María Lizarazo López}

Candidata a Magíster en Pedagogía de la Cultura Física

Universidad Pedagógica y Tecnológica de Colombia

Tunja, Colombia

laura.lizarazo01@uptc.edu.co

Orcid: https://orcid.org/0000-0003-2237-5455

\section{Margoth Adriana Valdivieso Miranda}

Magister en Ciencias-Estadística

Universidad Pedagógica y Tecnológica de Colombia

Tunja, Colombia

margoth.valdivieso@uptc.edu.co

Orcid: https://orcid.org/0000-0002-3617-928X

Recibido: 31 de octubre de 2019

Evaluado: 30 de marzo de 2020

Tipo de artículo: Investigación Científica y Tecnológica.

\author{
Víctor Miguel Ángel Burbano Pantoja \\ Doctor en Ciencias de la Educación \\ Universidad Pedagógica y Tecnológica de Colombia \\ Tunja, Colombia \\ victorburbanop@yahoo.es; victor.burbano@uptc.edu.- \\ co \\ Orcid: https://orcid.org/0000-0002-3561-1886
}

\section{Resumen}

En diversos contextos, la actividad física (AF) afecta el desarrollo integral de los adolescentes, puesto que genera múltiples beneficios a nivel físico y psicológico, en contraposición al sedentarismo que causa perjuicios en la salud de quienes lo padecen. El objetivo del trabajo investigativo consistió en estudiar las correlaciones existentes entre la AF y cuatro dimensiones en la autonomía de 90 escolares, con edades entre los 14 y los 17 años y pertenecientes a una institución educativa colombiana. La metodología abordada fue cuantitativa con diseño transversal, de tipología descriptiva-correlacional; la información se recolectó mediante el instrumento Physical Activity Questionnaire para adolescentes (PAQ-A) y el inventario de Coopersmith; los datos fueron procesados mediante el software libre. Según los resultados, la AF de dichos escolares mostró niveles relativamente bajos y se correlacionó de forma significativa, positiva y moderada con su autoestima en las dimensiones: social, general, hogar y escolar; 
asimismo, entre algunas de estas últimas. En conclusión, cerca del 50\% de los escolares presentó bajos niveles de autoestima, atribuibles a su poca ejecución de AF; en el resto, un incremento de la práctica de AF generó un aumento de su autoestima, por lo que se requiere promoverla desde la escuela y el hogar.

Palabras clave: Actividad física; Adolescencia; Desarrollo integral; Dimensiones autoestima, Nivel escolar.

\section{| Abstract}

In various contexts, physical activity (AP) affects the integral development of adolescents, since it generates multiple benefits at a physical and psychological level, as opposed to sedentary lifestyle that causes damage to the health of those who suffer from it. The objective of the research work was to study the correlations between the AF and four dimensions in the autonomy of 90 schoolchildren whose ages are from 14 to 17 years and belong to a Colombian educational institution. The methodology addressed was quantitative with a transversal design of descriptive-correlational typology; the information was collected through the Physical Activity Questionnaire instrument for adolescents (PAQ-A) and the Coopersmith inventory, the data was processed using free software R. According to the results, the PA of these schoolchildren showed relatively low levels and was significantly, positively and moderately correlated with their self-esteem in the dimensions: social, general, home and school; also, among some of the latter. In conclusion, about $50 \%$ of schoolchildren presented low levels of self-esteem attributable to their low performance of FA; in the rest, an increase in the practice of FA generated an increase in their self-esteem; that is why it is required to promote it from school and home.

Keywords: Physical activity; Adolescence; Integral development; Self-esteem dimensions; School level.

\section{| Introducción}

La educación es un proceso asociado con la formación de un sujeto, quien se construye en un contexto social afectado por la interacción con las demás personas, pero con las restricciones y exigencias establecidas en espacios como el hogar o la escuela (Jaramillo, Quilindo y Paz, 2017); sin embargo, la vida estudiantil va más allá de estos espacios, e involucra procesos de desarrollo, tanto físicos como de conductas pro sociales (Redondo, Luzardo, Larrotta y Rangel, 2015), los cuales están influenciados por la cultura imperante, tanto en el hogar como en la sociedad. En la vida cotidiana, el estudiante soporta una alta carga emocional, matizada por los problemas sociales que se generan en su entorno, tales como la violencia en sus distintas manifestaciones, los conflictos presentes en los diversos espacios de la escuela, el hogar y la sociedad, los desafíos que implica la convivencia escolar (Londoño, Estrada y Alvarado, 2018), entre otros. Estos problemas pueden desencadenar diferentes tipos de reacción en los adolescentes, como la depresión, la ideación suicida, el cyberbulling (Salas, Levette, Redondo y Luzardo, 2017), la agresividad escolar (Munevar, Burbano y Flórez, 2019), y la baja autoestima, solo por mencionar algunos. 
Por otra parte, desde diferentes miradas teóricas, se sugiere que los individuos a lo largo de la vida, en particular en la adolescencia, etapa donde el individuo establece su identidad personal (Salas et al., 2017), en presencia de cambios fisiológicos y mentales, se preocupan por proyectar y mantener una imagen positiva de sí mismos, y exteriorizar su forma de percibirse y auto valorarse en un ambiente específico (Franco, Coterón y Gómez, 2017) o en contextos poco explorados por ellos. Estos aspectos pueden potenciarse por medio de la interacción con grupos de similar bagaje cultural (Pérez y Smith, 2008) o a través de diversas estrategias que pueden incluir la participación de la escuela, el hogar y el entorno social; entre esas estrategias están: la realización de AF, el diálogo sobre el manejo de conflictos y el perdón como objeto cultural, y una acción contrapuesta a la agresión y demás formas de violencia (J. E. Vásquez y López, 2018). Sin embargo, no siempre es posible mantener una autoestima alta por los cambios fisiológicos, cognitivos, emocionales y sociales, propios de la adolescencia (M. Estévez et al., 2015).

En general, los adolescentes que presentan bajos niveles de autoestima (AE), tienden a caracterizarse por llevar una vida física poco activa, un riesgo más elevado de adquirir hábitos insalubres y una salud mental pobre (Orth, Trzesniewski \& Robins, 2010). Estos factores los hacen propensos a caer en conductas agresivas, sintomas de ansiedad, desesperanza, depresión y hasta tendencias suicidas (Zurita et al., 2017); en cuanto a las conductas agresivas, estas se pueden superar con la práctica de AF (Munevar et al., 2019).

Una forma de paliar los bajos niveles de AE presentes en algunos adolescentes, quienes interactúan con sus contemporáneos en las instituciones educativas, es la práctica frecuente de AF. Diversos estudios evidencian que la realización de AF, de manera regular, genera beneficios tanto en el aspecto físico como psicológico de los individuos (Akandere y Tekin, 2005); en particular, los adolescentes pueden mejorar su AE con la orientación de los docentes dentro de las aulas, de los familiares en el hogar y de las demás personas con las cuales interactúa en el medio que lo rodea; en este sentido Moreno, Cervelló y Moreno (2008) mencionan que la AE puede influenciarse por los núcleos sociales cercanos al adolescente y la práctica de la AF. En consecuencia, la AF se constituye en un aspecto que puede afectar el desarrollo integral en los adolescentes, puesto que genera múltiples beneficios a nivel físico y psicológico.

En este contexto, es pertinente mencionar que los profesores de educación física (EF) cuentan con un escenario propicio para diagnosticar y proponer acciones que coadyuven con una mejoría en la AE del estudiantado, de modo que también potencien el bienestar físico, emocional, personal y social del adolescente, favoreciendo con ello su formación integral y la apropiación de aspectos tendientes a conseguir una vida más saludable.

En concordancia con los elementos conceptuales que se exponen en la sección subsiguiente, en esta investigación se propuso explorar niveles de prevalencia de AF y su correlación con cuatro dimensiones de AE (personal o general, académica o escolar, social y familiar u hogar), en adolescentes escolarizados; esto implicó efectuar un diagnóstico y proponer programas de intervención para fortalecer la formación integral y el incremento de la AE de adolescentes, desde la clase de EF y del entorno escolar al cual pertenecen. Este trabajo investigativo buscó potenciar las acciones de los individuos, en el sentido de que la ejecución de AF ha de proporcionar una vida más saludable y menguar los bajos niveles de AE. 


\section{Elementos conceptuales}

En esta sección, de forma sucinta, se exponen los elementos conceptuales que soportaron los procesos investigativos, y se mencionan algunas investigaciones que sirvieron como antecedentes para fundamentar el objeto de estudio. En referencia a tales elementos, se abordan de manera secuencial: la actividad física (AF), la adolescencia, la autoestima (AE) y los antecedentes.

La AF se define a través del conjunto de movimientos del cuerpo, en el cual se hace un gasto de energía e involucra diversos músculos (Lizarazo, 2018); además, es una experiencia individual y personal que le permite al individuo relacionarse con otros seres y el medio que les rodea, por eso, puede realizarse en la escuela, el hogar y variedad de sitios, bajo normas regulativas o en los tiempos de ocio (Munevar et al., 2019); esta incluye actividades cotidianas, tales como caminar y oficios del hogar, actividades deportivas y competitivas, entre otras (Vidarte, Vélez, Sandoval y Mora, 2011). En concordancia con Zurita et al. (2017), la AF corresponde a la cantidad e intensidad de movimiento voluntario del cuerpo, requerida para asegurar un adecuado estado de salud; los movimientos incluidos en la misma implican un determinado gasto de calorías. La $A F$, también, es una forma de movimiento que permite al ser humano relacionar su mente, sus necesidades de expresión o interacción con otros o con el medio que lo rodea; incluso, ha de incluir rutinas de ejercicios corporales o la ejecución de diversas actividades en el ámbito del trabajo, el juego, el transporte, entre otros.

Según la Organización Mundial de la Salud -OMS- (2018), el abordaje de la AF desde la educación está ligado con algunas disciplinas científicas, naturales y humanas; por tanto, los procesos pedagógicos utilizados en esta disciplina contribuyen a la formación de personas, quienes ponen la AF a su disposición para satisfacer sus gustos y necesidades (Vidarte et al., 2011); en este sentido, la AF es una forma efectiva de intervención. Además, la AF posibilita el aumento de la autopercepción y de complacencia colectiva; por consiguiente, se constituye en una herramienta efectiva para lograr mejores formas de vivir en la población mundial, promocionar la salud, modificar hábitos y mejorar comportamientos en la interacción social.

Por otro lado, la adolescencia se conceptualiza como un periodo de la vida del ser humano en la cual crece y se desarrolla, tanto biológica como culturalmente; ocurre entre la niñez y adultez, y puede ubicarse entre 10 y 15 años, en dependencia con cierto contexto; la adolescencia es un periodo caracterizado por la presencia de diversos cambios, tanto en lo físico como en lo intelectual (OMS, s.f.); y el establecimiento de la identidad, al alejarse de sus ideas infantiles e interactuar con los adultos (Salas et al., 2017). En esta etapa, el individuo presenta fluctuaciones en su estado de ánimo, autoestima, maneras de pensar, formas de actuar, apariencia personal, entre otras; algunas veces se involucra en problemas de tipo conductual (por ejemplo, agresión), emocional, social o alimentario (Echeverri, 2015). Aquí la AF cumple un papel fundamental aportando múltiples beneficios, tales como el desarrollo de tres sistemas: locomoción, neuro-muscular y cardio-vascular; el control de la depresión y de la ansiedad; un mejor rendimiento escolar; y la generación de comportamientos salubres (OMS, s.f.).

La AE corresponde a un juicio de valor, una autoevaluación que el individuo posee de sí 
mismo y se manifiesta hacia a otros por medio de acciones específicas o expresiones verbales, esta puede ser tanto positiva como negativa, y expresada a través de sus actitudes (Mesías, 2017); en otras palabras, la AE le permite al individuo percibirse y auto valorarse en un contexto específico, mantener y proyectar una imagen de sí mismo ante otras personas (Franco et al., 2017), compararse con otros, desarrollar patrones y reconocerse como persona; por consiguiente, puede incluir tanto elementos personales como sociales (E. Estévez, Martínez y Musitu, 2006), e implica factores preceptúales y cognitivos (Alcantará, 2007).

Además, las dimensiones de la AE son susceptibles de determinarse por medio de la batería de Coopersmith (1976), ya que cada individuo reacciona de manera distinta ante situaciones semejantes, generándose diferentes expectativas hacia el futuro, acciones, reacciones y auto conceptos diversos (Mesías, 2017). Asimismo, en los trabajos de Guillón, Crocq \& Bailey (2007), Mullis \& Chapman (2000), y Pacheco, Luarte, Pérez y Linz (2015), se aplica tal inventario para determinar las relaciones de la AE con diversos tipos de variables. Sin duda los investigadores interesados en estudiar la $\mathrm{AF}$, reconocen que se producen variados tipos de correlaciones entre esta y la autoestima. Por otra parte, la AF ayuda a promover la salud, prevenir enfermedades y generar beneficios, tanto mentales como psicológicos; asimismo, contribuye con la mengua de la agresividad en sus diferentes manifestaciones (Munevar et al., 2019).

En cuanto a los antecedentes, el problema de la AE y la AF ha sido investigado ampliamente desde diversas miradas teóricas y en diferentes contextos, por tratarse de un asunto neurálgico en el crecimiento del ser humano; en el ámbito internacional, los estudios realizados por Schneider, Fridlund \& Cooper (2008), Dishman, Felton, Hales \& Sward (2006), y Schmalz, Deane \& Birch (2007) sugieren que la AF se asocia de forma positiva y moderada con la autoestima, bajo determinadas circunstancias; es decir, un incremento en la AF puede producir un mayor nivel de $\mathrm{AE}$, ligada a una percepción más positiva de imagen corporal. Además, es posible que la realización de AF se vincule directamente con la participación del individuo en los deportes, lo cual puede generar una mayor AE, ligada al incremento del auto-concepto de tipo físico (Dishman et al., 2006). En referencia con la AF, la AE y la adolescencia, en una revisión sistemática realizada por Biddle \& Asare (2011), se establece que, a corto plazo, la AF originó efectos positivos sobre la AE de ciertos individuos; por tanto, aquellos que son físicamente activos poseen una menor probabilidad de atravesar problemas referidos a su salud mental (Amoedo y Juste, 2016).

Adicionalmente, en Murcia-España ha sido de interés establecer correlaciones que pueden ocurrir entre la ejecución frecuente de AF, la ansiedad, el auto concepto y la depresión, en adolescentes con género femenino (Campillo, Zafra y Redondo, 2008); los resultados mostraron que las participantes, quienes realizaron AF frecuentemente, obtuvieron puntuaciones superiores en el auto concepto, y disminuyeron tanto su ansiedad como su depresión. También, se han analizado los efectos de la AF sobre la salud física y mental de jóvenes ingleses (Fairclough, \& Stratton, 2005); encontrándose que, la poca práctica de AF durante la niñez afecta el desarrollo esquelético-muscular en la vida posterior; en cambio, una ejecución vigorosa contribuye con la mejora de la composición física y los aspectos emocionales del adolescente, en particular de su AE. Por otro lado, en Lima-Perú, Montoya y Pinto (2016) determinaron niveles de AF en correspondencia con el instrumento PAQ-A, el cual fue aplicado en alumnos de 
educación secundaria; con los resultados se estableció que alrededor del $76 \%$ de los adolescentes presentaron niveles bajos de AF, los cuales afectaban su desempeño académico y su percepción de sí mismos.

De otro lado, diversos aspectos de la AF, la obesidad y la AE fueron explorados en escolares chilenos (Zurita et al., 2017); los hallazgos reflejaron que la obesidad se asocia con una baja AE, y existe una relación positiva y moderada de la AF con la AE. También, en la Universidad de Málaga, se hace una revisión investigativa sobre la AE en escolares adolescentes (Rodríguez y Caño, 2012); los trabajos revisados sugirieron la necesidad de intervenir a quienes presentaran una baja AE. Adicionalmente, Pacheco et al. (2015) aplicaron un programa de intervención y concluyeron que tal programa sí aumentó la AE de un grupo de alumnos chilenos. También, sobre escolares de Chile, Delgado, Bastian, Jerez, Cofré y Martínez (2019) establecieron la existencia de correlaciones moderadas entre la obesidad, el sobrepeso y la AE en diferentes niveles. En Costa Rica, M. F. Herrera et al. (2019) concluyeron que existía una relación positiva entre AF y AE; en cambio, entre el sobrepeso y la $A E$, tal relación fue negativa en alumnos del ciclo II del Cantón Central de Heredia. Por su parte, en España, Pérez, Aznar, Nuviala \& Nuviala (2019), mediante un trabajo de revisión, determinaron que la AF se constituía en una estrategia eficiente para la promoción de la salud en escolares de secundaria. Finalmente, en Honduras, A. A. Vásquez, Zelaya y García (2019), por medio de un análisis factorial, hallaron una correlación positiva muy alta entre la AE y la AF; en contraste, el sobrepeso afectaba de forma negativa y significativa a la AE en estudiantes de San Pedro Sula.

En el contexto colombiano, en Barranquilla, Borda et al. (2016), mediante un estudio de tipo descriptivo-transversal, establecieron que una alta AF actuaba como un factor protector de AE, y la percepción de la imagen corporal correlacionaba positivamente con el estado emocional y nutricional de un grupo de escolares; aunque la AF practicada aún podía considerarse baja. En Caldas-Antioquia, Gallego, Medina y Montoya (2008), por medio de un estudio descriptivo de corte transversal e histórico, establecieron que el abuso sexual está asociado positivamente con una baja AE y deja secuelas de tipo emocional, relacionadas con la depresión y el consumo de sustancias psicoactivas en alumnos de educación media. Por su parte, Vidarte et al. (2011), a través de un trabajo de revisión sobre AF, argumentaron que es perentorio diseñar y ejecutar programas de intervención basados en $\mathrm{AF}$, a fin de promover la salud y autopercepción de los individuos en diversos rangos de edad, y combatir el sedentarismo, en particular durante la adolescencia.

De otro lado, Piñeros y Pardo (2010) aplicaron el instrumento denominado "Encuesta mundial de salud a escolares" en cinco ciudades de Colombia, y hallaron porcentajes bajos de AF en adolescentes entre 13 y 15 años (9\% a 20\%), los cuales resultaron similares a las de otros países donde se habían aplicado tales encuestas; así las cosas, concluyeron que los estudiantes eran altamente sedentarios y, en consecuencia, se requería implementar programas de AF de forma urgente para promover su práctica y disminuir las factores de riesgo para la salud de los adolescentes, tanto hombres como mujeres. Finalmente, Munevar et al. (2019) determinaron ciertos efectos generados por un programa de AF sobre la agresividad escolar. Trabajos investigativos como los mencionados proporcionan elementos metodológicos para diagnosticar, a tiempo, la relación entre la AF y los niveles de AE de adolescentes en contextos específicos, a fin de generar reflexión docente que posibilite la implementación oportuna de programas de intervención. 


\section{| Metodología}

En esta sección se indica el enfoque investigativo abordado, los escolares quienes participaron en el estudio, los instrumentos, procedimientos y la forma cómo se hizo el análisis estadístico.

\section{Enfoque investigativo}

Este trabajo fue desarrollado desde un enfoque cuantitativo, y el diseño fue transversal con tipología descriptiva y correlacional (Hernández, Fernández y Batista, 2003); en los estudios de corte transversal la información se recolecta en un momento específico del tiempo, sin requerirse tomarla nuevamente, como sí ocurre en los diseños cuasi-experimentales. En este contexto, se analizó las correlaciones entre cuatro dimensiones de AE y la realización de AF de 90 adolescentes escolarizados, cuyas edades están entre los 14 y los 17 años, y pertenecen a una institución educativa ubicada en el departamento de Boyacá, en Colombia.

\section{Participantes}

La población estuvo constituida por 450 alumnos matriculados en educación secundaria en una institución educativa; el número de participantes que conformó la muestra se obtuvo usando un 95\% ( $Z=1.96)$ de confianza, trabajándose bajo el criterio de total incertidumbre, con $p=0.5, q=0.5$, y se asumió un error absoluto de e=9.3\%; se estableció que el tamaño muestral era de 90 estudiantes. Se efectuó un muestreo por conveniencia, atendiendo a las características del instrumento PAQ-A y Coopersmith, destinados a recoger los datos; además, se tuvo en cuenta: la AF a desarrollar en el contexto de la clase de EF, y los criterios sobre inclusión (estar con matrícula activa en el grado noveno, contar con el consentimiento de sus padres y estar en capacidad de realizar AF).

\section{Instrumentos}

En este trabajo se aplicó la versión española del PAQ-A, un instrumento construido para medir AF en adolescentes a través de puntuaciones, las cuales van desde un nivel muy bajo hasta muy intenso, en cuanto a la AF ejecutada en los siete últimos días (Martínez et al., 2009; Mesías, 2017). El PAQ-A se constituye por nueve preguntas, las cuales permiten valorar diferentes aspectos de la AF en distintos momentos de cada día observado; se puntúa con un número entero desde 1 hasta 5, número asociado con un nivel de AF desarrollado por cada participante (Mesías, 2017). El PAQ-A proviene del PAQ-C destinado a niños mayores, el cual ha sido modificado para que se pueda aplicar a estudiantes de educación secundaria (Kowalski, Crocker \& Kowalski, 1997). En este trabajo, con antelación, se aplicó el PAQ-A a una muestra piloto (de estudiantes de otro colegio, con características similares a quienes participaron en el presente estudio), obteniéndose un alpha de Crombach del 0.808; en la muestra de este estudio se obtuvo una confiabilidad de 0.82, similar al obtenido por Lizarazo (2018). 
De otro lado, la batería de Coopersmith permite medir la autoestima de forma cuantitativa; esta fue construida con cincuenta ítems en 1959, los cuales valoran las percepciones de cada individuo, y se organizan en 4 áreas denominadas: sí mismo, sobre sus pares, el colegio y los padres (Brinkmann, Segure y Solar, 1989; Mesías, 2017); esta batería en su formato "A" se validó en Chile, lográndose una confiabilidad de 0.92. Respecto a tal batería, Prewitt (1984) menciona que se trata de un auto reporte que posibilita valorar la percepción estudiantil por medio de 50 ítems, organizados en 4 dimensiones o áreas llamadas: autoestima social, general, padres y hogar (Lizarazo, 2018; Mesías, 2017), y para las cuales se obtuvo una confiabilidad de 0.814, 0.783, 0.811 y 0.806 , respectivamente, en la muestra de 90 participantes.

\section{Procedimiento}

En cuanto al procedimiento, en principio fue necesario hablar con las directivas del colegio, con el propósito de socializar el proyecto de investigación y dialogar sobre los mecanismos tendientes a obtener un consentimiento (informado), firmado por los respectivos padres, y el asentimiento de los escolares adolescentes, quienes decidieron participar de manera voluntaria en el estudio; se aclaró que la información recogida se usaría exclusivamente con fines académicos e investigativos, y se manejaría de manera confidencial, en consonancia con el código ético, y las normas constitucionales y legales colombianas sobre protección de datos personales (Ley 1266 de 2008 (Habeas data) y la Ley 23 de 1982). Con los permisos ya obtenidos, se dialogó con los participantes sobre las actividades inherentes y la aplicación de los cuestionarios durante el tiempo de clase en la asignatura de EF (Mesías, 2017). Se inició con un diagnóstico de $\mathrm{AE}$, en el estudiantado participante en el proyecto, por medio de la batería Coopersmith, valorándose las dimensiones: general, escolar, hogar y social. Luego, se midió los niveles de AF a cada participante, por medio del PAQ-A; las puntuaciones obtenidas por los alumnos permitieron organizar 5 niveles: muy bajo, bajo, moderado, intenso, y muy intenso (Lizarazo, 2018). En cuanto al trabajo de campo desarrollado y a la forma cómo se recolectaron los datos, hubo un riesgo bajo, tanto en la integridad como en la salud de los participantes en el estudio.

\section{Procesado de datos}

El procesado de datos se hizo por medio del software libre R. Se elaboró un análisis para cada variable, una correspondiente a los niveles de AF y la otra a las dimensiones de la AE; luego, se determinaron las correlaciones respectivas. Para esto, se calculó la matriz de correlaciones, en la que los elementos ubicados fuera de la diagonal corresponden al coeficiente $r$ de Pearson, el cual se obtiene con los datos de las puntuaciones, recogidos mediante los dos instrumentos mencionados. Un valor r próximo a 1 es indicativo de que existe una correlación positiva y fuerte; en cambio, si r se acerca a -1, la correlación será negativa y fuerte; cuando $r$ está cercano a 0.5 la correlación se considera moderada y positiva; por el contrario, si r está próximo a -0.5, la correlación será negativa y moderada; finalmente, si r está cerca de cero, ya sea por la derecha o por la izquierda, la correlación será positiva y débil, o negativa y débil, respectivamente (Burbano y Valdivieso, 2016). 


\section{| Resultados}

Inicialmente, se indican los resultados obtenidos; para ello, se presentan algunas tablas y se realiza una exégesis o interpretación de los datos procesados, cuya información provino de la evidencia empírica obtenida por medio del PAQ-A y el cuestionario de Coopersmith; se inicia generando una clasificación por niveles de AF, luego se prosigue con los correspondientes a la $A E, y$, por último, se explicita la correlación calculada para estas dos variables.

\section{Clasificación por niveles de AF}

En correspondencia con el PAQ-A, se organizaron cinco niveles de AF: "muy bajo", "bajo", "moderado", "intenso", y "muy intenso" (Mesías, 2017), en concordancia con los siguientes rangos de puntuación promedio: 1-1,49; 1,50-2,49; 2,50-3,49; 3,50-4,49 y 4,50-5,0; estos rangos permitieron clasificar a los participantes del estudio, en consonancia con los cálculos que se pueden observar en la tabla 1, de la cual se deduce que: 2 (2.2\%) de los escolares se clasificaron en un nivel muy bajo de AF, 51 (56.6\%) en el nivel bajo, 29 (32.2\%) en el nivel moderado, 7 (7.7\%) en intenso, y solamente 1 (1.1\%) en el nivel muy intenso. Esta clasificación evidencia que existe una tendencia a que los adolescentes se ubiquen en el nivel bajo, seguido del nivel moderado, con un porcentaje acumulado del $88,8 \%$; en menor porcentaje le siguieron los del nivel intenso, muy bajo y muy intenso, respectivamente.

\section{Tabla 1}

\section{Clasificación por niveles de $A F$}

\begin{tabular}{llccc}
\hline & & Frecuencia & Porcentaje & Porcentaje acumulado \\
\cline { 1 - 3 } Niveles & Muy bajo & 2 & $2,2 \%$ & $2,2 \%$ \\
& Bajo & 51 & $56,6 \%$ & $58,8 \%$ \\
& Moderado & 29 & $32,2 \%$ & $91 \%$ \\
& Intenso & 7 & $7,7 \%$ & $98,8 \%$ \\
& Muy intenso & 1 & $1,1 \%$ & $100 \%$ \\
& Total & 90 & $100 \%$ & \\
\hline
\end{tabular}

Nota: elaboración propia, a través del paquete $\mathrm{R}$

\section{Clasificación para autoestima}

La batería de Coopersmith hizo posible clasificar a los adolescentes por el puntaje total de $\mathrm{AE}$, en cinco categorías o niveles: "muy bajo", "bajo", "normal”, "alto", y "muy alto" (Lizarazo, 2018; Mesías, 2017), en correspondencia con los rangos establecidos en el mencionado inventario. Los escolares participantes en el estudio se clasificaron como se indica en la tabla 2. 


\section{Tabla 2}

Clasificación por puntaje total de autoestima

\begin{tabular}{llccc}
\hline & & Frecuencia & Porcentaje & Porcentaje acumulado \\
\hline \multirow{5}{*}{ Niveles } & Muy bajo & 8 & $8,8 \%$ & $8,8 \%$ \\
& Bajo & 14 & $15,5 \%$ & $24,3 \%$ \\
& Normal & 45 & $50 \%$ & $74,3 \%$ \\
& Alto & 17 & $18,8 \%$ & $93,1 \%$ \\
& Muy alto & 6 & $6,6 \%$ & $100 \%$ \\
& Total & 90 & $100 \%$ & \\
\hline
\end{tabular}

Nota: elaboración propia, a través del paquete $\mathrm{R}$.

Con la evidencia empírica recolectada, mediante el inventario de Coopersmith, también se consolidaron cuatro dimensiones para la autoestima, las cuales fueron: general (AE_general), social (AE_social), escolar (AE_escolar) y hogar (AE_hogar). En referencia con la clasificación por puntaje total de autoestima, con base en la tabla 2, se determinó que prevalece el nivel normal con un $50 \%$, seguido del nivel alto de autoestima (18,8\%); estos dos niveles generan un porcentaje acumulado del 68,8\%; en menor porcentaje le siguieron los estudiantes quienes se clasificaron en el nivel bajo (15.5\%), muy bajo (8.8\%) y muy alto (6.6\%), respectivamente. Estos resultados permiten interpretar que los estudiantes del grupo presentan una tendencia a ubicarse en el nivel normal de AE; sin embargo, también se hacen visibles niveles bajos de autoestima y poca prevalencia de niveles altos.

En lo que respecta a las dimensiones de la $A E$, se estableció que: i) en la autoestima general, el grupo presenta una tendencia a ubicarse en el nivel normal (46,2\%), seguido por los niveles bajo (20,9\%), alto (19,8\%), muy bajo (12,1\%) y muy alto (1,1\%); ii) en la autoestima social, el grupo tiende a ubicarse en el nivel normal (50,5\%), seguido por los niveles muy bajo $(20,9 \%)$, bajo $(20,9 \%)$ y alto $(7,7 \%) ; \mathrm{iii})$ en la autoestima escolar, el grupo tiende a ubicarse en el nivel normal $(45,1 \%)$, seguido por los niveles alto $(27,5 \%)$, muy alto (17,6\%), muy bajo $(6,6 \%)$ y bajo $(3,3 \%)$; iv) en la autoestima hogar, el grupo tiende a ubicarse en el nivel normal (41,8\%), seguido por los niveles bajo (18,7\%), muy bajo (16,5\%), alto $(15,4 \%)$ y muy alto $(7,7 \%)$. Estos porcentajes indican que, con frecuencia, en la dimensión general los participantes manifiestan actitudes positivas, aspiraciones, confianza en sí mismos, buenos atributos personales y deseos por mejorar; en la dimensión social, se muestran seguros y con habilidades para comunicarse y adaptarse a nuevos amigos en diferentes contextos; en la dimensión escolar, tienen personalidad para enfrentar, con relativo éxito, sus estudios mediante el uso de sus capacidades intelectuales y creativas; y en la dimensión hogar, se sienten aceptados y apoyados por el vínculo familiar.

\section{Análisis correlacional}

Se determinó una correlación general entre el total de las puntuaciones referidas a la AF y el puntaje total de $A E$, que provienen de acumular los puntajes del cuestionario PAQ-A y de la batería de Coopersmith, respectivamente. Posteriormente, se presentan e interpretan las 
correlaciones que se calcularon entre la AF y las cuatro escalas de autonomía en los adolescentes.

Respecto a la AF y la autoestima general, se estableció que existe una correlación (general) significativa al $5 \%(0,05)$, entre las puntuaciones del PAQ-A y las del inventario de CooperSmith, puesto que se obtuvo un p_valor $=0.000$, el cual es inferior a 0.05; además, se obtuvo un $r=$ 0,$434 ; p<0,05 ; 2$ _colas (2-tailed). Por consiguiente, al interpretar el coeficiente $r$ se concluye que hay una correlación significativa, positiva y moderada entre las mencionadas puntuaciones.

Tabla 3

Correlaciones entre $A F$ y escalas de autoestima ( $A E)$

\begin{tabular}{|c|c|c|c|c|c|c|}
\hline & & $\begin{array}{l}\text { AF- } \\
\text { Total }\end{array}$ & $\begin{array}{c}\text { AE- } \\
\text { General } \\
\end{array}$ & $\begin{array}{c}\text { AE- } \\
\text { Social }\end{array}$ & $\begin{array}{c}\text { AE- } \\
\text { Escolar }\end{array}$ & $\begin{array}{c}\text { AE- } \\
\text { Hogar }\end{array}$ \\
\hline \multirow[t]{2}{*}{ AF-Total } & $\begin{array}{l}\text { Correlación } \\
\text { Pearson }\end{array}$ & 1 & 0,316 & 0,297 & 0,328 & 0,210 \\
\hline & $\begin{array}{l}\text { Sig (2-tailed } \\
\mathrm{N}\end{array}$ & 90 & $\begin{array}{r}0,002^{*} \\
90\end{array}$ & $\begin{array}{r}0,004^{*} \\
90\end{array}$ & $\begin{array}{r}0,002^{*} \\
90\end{array}$ & $\begin{array}{r}0,046^{*} \\
90\end{array}$ \\
\hline \multirow[t]{2}{*}{$\begin{array}{l}\text { AE- } \\
\text { General }\end{array}$} & $\begin{array}{l}\text { Correlación } \\
\text { Pearson }\end{array}$ & 0,316 & 1 & 0,444 & 0,465 & 0,267 \\
\hline & $\begin{array}{l}\text { Sig (2-tailed } \\
\mathrm{N}\end{array}$ & $\begin{array}{r}0,002 \\
90\end{array}$ & 90 & $\begin{array}{r}0,000^{*} \\
90\end{array}$ & $\begin{array}{r}0,000^{*} \\
90\end{array}$ & $\begin{array}{r}0,011^{*} \\
90\end{array}$ \\
\hline \multirow[t]{2}{*}{ AE-Social } & $\begin{array}{l}\text { Correlación } \\
\text { Pearson }\end{array}$ & 0,297 & 0,444 & 1 & 0,391 & 0,154 \\
\hline & $\begin{array}{l}\text { Sig (2-tailed } \\
\mathrm{N}\end{array}$ & $\begin{array}{r}0,004 \\
90\end{array}$ & $\begin{array}{r}0,000 \\
90\end{array}$ & 90 & $\begin{array}{r}0,000^{*} \\
90\end{array}$ & $\begin{array}{r}0,145 \\
90\end{array}$ \\
\hline \multirow[t]{2}{*}{ AE-Escolar } & $\begin{array}{l}\text { Correlación } \\
\text { Pearson }\end{array}$ & 0,328 & 0,465 & 0,391 & 1 & 0,190 \\
\hline & $\begin{array}{l}\text { Sig (2-tailed } \\
\mathrm{N}\end{array}$ & $\begin{array}{r}0,002 \\
90\end{array}$ & $\begin{array}{r}0,000 \\
90\end{array}$ & $\begin{array}{r}0,000 \\
90\end{array}$ & 90 & $\begin{array}{r}0,071 \\
90\end{array}$ \\
\hline \multirow[t]{2}{*}{ AE-Hogar } & $\begin{array}{l}\text { Correlación } \\
\text { Pearson }\end{array}$ & 0,210 & 0,267 & 0,154 & 0,190 & 1 \\
\hline & $\begin{array}{l}\text { Sig (2-tailed } \\
\mathrm{N}\end{array}$ & $\begin{array}{r}0,046 \\
90\end{array}$ & $\begin{array}{r}0,011 \\
90 \\
\end{array}$ & $\begin{array}{r}0,145 \\
90 \\
\end{array}$ & $\begin{array}{r}0,071 \\
90\end{array}$ & 90 \\
\hline
\end{tabular}

Nota: Elaboración propia, a través del paquete R. * las correlaciones son significativas al $5 \%$ de significancia. 
De la tabla 3, se tienen diez correlaciones, las cuales se generan entre las puntuaciones totales de la actividad física (AF-Total) y las puntuaciones de las dimensiones AE_general, AE_social, AE_escolar y AE_hogar, de las cuales, ocho fueron significativas al $5 \%(0,05)$ y dos son no significativas. En seguida, se describen las correlaciones entre la AF-Total y las cuatro dimensiones.

Entre la AF y la autoestima general existe una correlación significativa ( $p \_$valor $=0,002 ; r=$ 0,$316 ; p<0,05 ; 2$ colas (2-tailed)). Al interpretar el valor de $r$ se establece la presencia de una relación moderada y positiva, la cual indica que un aumento en la AF implica un moderado incremento en las puntuaciones de la autoestima general, y viceversa. Asimismo, entre la AF_total y la AE_social se presenta una correlación significativa ( $p \_v a l o r=0,004 ; r=0,297$ ). El valor de $r$ indica que existe una correlación positiva y moderada; por consiguiente, un aumento en la AF implica un moderado incremento en las puntuaciones de la AE_social, y viceversa.

Asimismo, entre la AF y la autoestima escolar se presenta una correlación significativa ( $p \_v a l o r=0,002 ; r=0,328$ ); el valor de $r$ indica que existe una correlación positiva y moderada; así entonces, un aumento en la AF implica un moderado incremento en las puntuaciones de la de $A E_{-}$escolar, y viceversa. Entre la AF y la AE_hogar y padres existe una correlación significativa ( $p \_v a l o r=0,046 ; r=0,210$ ); este valor de $r$ refleja la existencia de una relación positiva y débil, indicando que un aumento en la AF produce un aumento débil en la AE_hogar. En este sentido, los padres han de ser conscientes de que pueden influir positivamente en el desarrollo de sus hijos; por tanto, han de inducirlos hacia la práctica más frecuente de AF, con independencia en lo referente a sus carencias, particularidades o potencialidades.

También existen correlaciones significativas entre la $A E$ general, con respecto a la AE social, escolar y hogar; además, entre la AE_social y la AE_escolar. Finalmente, entre la AE_social y $A E \_$hogar existe una correlación positiva y débil, la cual resultó no significativa; además, entre

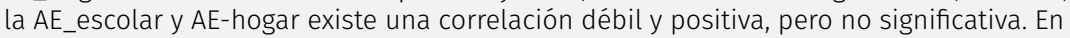
seguida, se indican las cuatro correlaciones significativas mencionadas. Entre la AE_general y

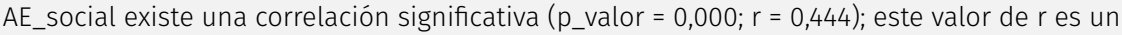
indicativo de que hay una correlación positiva y moderada.

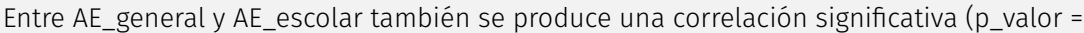
$0,000 ; r=0,465)$; luego existe una correlación positiva y moderada, la cual indica que un

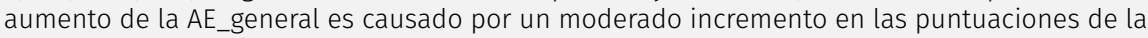
autoestima escolar, y viceversa. Entre la $A E \_$general y la $A E \_$hogar y padres se da una correlación significativa ( $p \_v a l o r=0,011 ; r=0,267$ ); así entonces, existe una correlación positiva y moderada, la cual refleja que un aumento de la $A E \_$general implica un moderado incremento

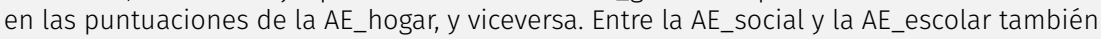
existe una correlación significativa ( $p \_v a l o r=0,000 ; r=0,391$ ); luego, hay una correlación positiva y moderada. En consecuencia, un aumento de la AE_social es atribuible a un aumento moderado de los puntajes en la AE_escolar, y al contrario. 


\section{| Discusión}

El propósito de este trabajo fue el de explorar los niveles de AF realizados por los adolescentes de noveno grado, de un colegio ubicado en un contexto particular, caracterizado por ubicarse en una zona andina en Colombia; asimismo, analizar las correlaciones entre la AF y la AE_general, AE_social, AE_escolar y AE_hogar. Al respecto, los hallazgos han permitido diagnosticar que los participantes presentan bajos niveles de AF en su contexto natural, información que contrasta con la de otros trabajos efectuados, tanto a nivel internacional como nacional; por ejemplo, los de Campillo et al. (2008), Akandere y Tekin (2005), Fairclough \& Stratton (2005), Piñeros y Pardo (2010), respectivamente. En referencia con la $A E$, se encontró que de un $42 \%$ hasta un $51 \%$ de los escolares adolescentes se clasificaron en la categoría de normal en su $A E \_$general, $A E \_s o c i a l, A E \_$_escolar o $A E \_$hogar; estos resultados proporcionan evidencia empírica de que un buen porcentaje presenta niveles bajos en ciertas dimensiones de AE (Delgado et al., 2019; E. Estévez et al., 2006), y por consiguiente es perentorio intervenirlos; por otro lado, un porcentaje reducido de escolares tiene un alto nivel de $\mathrm{AE}$, hecho que conviene mantener por medio de estrategias motivacionales y con ejecución de AF, ya que esta se convierte en un factor protector de AE (Borda et al., 2016). Así entonces, la investigación tiene un valor agregado, en el sentido de que ha generado información adicional sobre el estado de la realización de AF y de AE en los escolares que participaron en este estudio; esta información es un reflejo potencial de la situación que pueden estar viviendo un buen número de colegios similares, en torno a este tema (Pérez et al., 2019); hecho que también ameritaría ser investigado en un futuro cercano.

Como producto de este trabajo se tienen las siguientes implicaciones prácticas: se propone formular programas de intervención con base en la realización frecuente de AF, acordes a las necesidades de afecto y formación integral de los adolescentes, e impulsadas tanto desde el colegio como del hogar, o a través de proyectos de aprovechamiento del tiempo libre; es decir, que se sugiere aumentar la realización de AF desde la clase de EF, pero se ha de continuar con un esfuerzo mancomunado con el hogar y el entorno social, a fin de generar una mejoría en quienes presentan bajos niveles de AE; también, la AE puede acrecentarse con una adecuada intervención docente, desde las demás materias o asignaturas que conforman el currículo escolar; además, es clave la participación, tanto del colegio como del hogar, en la formación emocional, social y cognitiva de los escolares adolescentes; por eso, es conveniente mejorar el diálogo e interacción pacífica hogar-colegio entre los distintos actores que intervienen en el proceso educacional; no solo conviene mejorar las diversas manifestaciones de AE, sino también disminuir la agresividad escolar y otras manifestaciones de violencia e ideas suicidas, por medio de la realización cotidiana de AF. Finalmente, el diagnóstico realizado al grado noveno se debe extender a los demás grados de este colegio, y en la medida de lo posible a otros del contexto regional y nacional (Delgado et al., 2019; Piñeros y Pardo, 2010); asimismo, desde el colegio y el hogar resulta perentorio efectuar un seguimiento a los adolescentes que presenten baja autoestima o realizan poca $A F$, a fin de intervenirlos oportunamente ( $M$. Estévez et al., 2015) con diversas estrategias.

Por otro lado, aunque las instituciones educativas realizan acciones tendientes a conseguir un aumento de AF en sus estudiantes, y con frecuencia los profesores la promueven en la clase de EF, en referencia a la AF los niveles alcanzados por los adolescentes son relativamente bajos; 
así lo evidencia el presente estudio, cuyos resultados son levemente mejores a los presentador por Montoya y Pinto (2016), quienes determinaron que un 75.9\% de su muestra se encontraba entre los niveles muy bajo y bajo de AF; de este 75.9\%, un 49.1\% correspondía al nivel bajo. Estos resultados evidencian la necesidad de dinamizar, con mayor compromiso docente y estudiantil, las actividades propuestas en los planes curriculares en EF, a nivel institucional, de modo que posibiliten acrecentar la ejecución de AF y, en consecuencia, la AE del adolescente; las clases bien planeadas y orientadas hacia el crecimiento personal del estudiante pueden coadyuvar con un desarrollo de actividad moderada y vigorosa en el adolescente, la cual también puede acrecentarse con la colaboración decidida de los padres, para que sus hijos realicen AF durante el tiempo extra clase; estos aspectos concuerdan con lo expuesto por Piñeros y Pardo (2010) y Fairclough \& Stratton (2005), respecto a los beneficios de hacer AF de manera frecuente.

En cuanto a las puntuaciones totales sobre $A E$, el grupo de participantes obtuvo un promedio de $29.92 \pm 6.203$, donde la cantidad 6.203 corresponde a la desviación estándar; lo anterior, indica que la AE también es relativamente baja y atribuible, en gran medida, a niveles relativamente bajos de AF, que presentan los estudiantes que participaron en este estudio; este promedio resultó también inferior a $52.54 \pm 10,19$, en el estudio realizado por Delgado et al. (2019); en ambos estudios, las percepciones de los participantes y la influencias de sus creencias influyen sobre la posibilidad de dedicar más tiempo al desarrollo de AF, en cambio, se ocupan en actividades de ocio, focalizadas en los videojuegos o la televisión, entre otras. En contraposición, quienes logran altos niveles de AE disfrutan de más experiencias positivas, tanto en la ejecución de AF como en otros ámbitos escolares (Rodríguez y Caño, 2012); de allí que se requiere impulsar, desde el colegio y el hogar, la práctica frecuente de AF, lo que conlleva a una mejoría en tales niveles. Así la cosas, se recomienda empezar con un diagnóstico adecuado que se puede realizar en la clase de EF, por medio de instrumentos confiables, para luego implementar programas de intervención que impulsen tanto su éxito académico como personal y social, y consecuentemente, un acrecentamiento de su AE (Pacheco et al., 2015).

Los hallazgos del presente estudio reflejaron que, cerca del $40 \%$ de los participantes presentaron niveles bajos o muy bajos en tres dimensiones de la autoestima: AE_general, AE_social y AE_Hogar; en cambio, casi un $10 \%$ de ellos se clasificó en esos mismos nivéleles en la cuarta dimensión denominada AE_escolar; estos porcentajes guardan similitud con los descritos por Pope, McHale \& Craighead (1988) en cuanto a los porcentajes obtenidos en su trabajo. También, estos resultados son comparables con los obtenidos por Bernal (2006), en términos de percentiles referidos al autoconcepto (autoestima) asociado con AE_general, AE_social, $A E_{\text {_escolar }}$ (académica) y AE_hogar (familiar), con ligeras variaciones.

Con fundamento en los resultados de la correlación general, se evidenció la existencia de una correlación positiva, significativa y moderada entre la $A F$ y la $A E$, la cual resultó levemente inferior al valor obtenido $(r=0.503)$ por Zurita et al. (2017); por consiguiente, la AF sí beneficia a los individuos en la mejora de su AE; estos resultados son concomitantes con lo manifestado por la OMS (2010). En esta misma dirección, la AF se relaciona con aspectos de tipo psicológico tendientes a una mejoría, en cuanto al control de la depresión, de la ansiedad juvenil y del desarrollo socio-emocional de los procesos educativos del estudiante (Barrios, Peña y Cifuentes, 2019); estos aspectos también están asociados con los hallazgos de este trabajo, los cuales indican que un incremento en la AF genera un moderado aumento en la AE; además, una mejora en la AE se constituye en un agente motivador para que los individuos acrecienten su práctica de 
AF. Adicionalmente, los resultados concuerdan tanto con los trabajos efectuados por Dishman et al. (2006) como por Schneider et al. (2008), quienes sostienen que la AF está asociada con la AE de forma moderada y positiva, generando resultados positivos en torno a la última.

En este trabajo, también se ha establecido que hay una correlación positiva, significativa y moderada entre la AF y las dimensiones de la AE en los escolares; esto es indicativo de que la ejecución de AF produce beneficios observables en un aumento de la AE estudiantil. En este sentido, Delgado et al. (2019) señalan que la AF presenta beneficios importantes en la regularización del peso corporal de escolares obesos y, en consecuencia, genera un aumento en su autoestima.

La presencia de una correlación positiva, significativa y moderada entre la AF y las dimensiones de AE_general y AE_social, confirma lo dicho por la OMS (2010), acerca de que la AF puede contribuir con su crecimiento socio-emocional, que habilita al adolescente para expresarse con más confianza. Los hallazgos en este estudio guardan relación con el trabajo realizado por Vidarte et al. (2011), en tanto que la AF se constituye en una manera efectiva de intervención, la cual hace posible el aumento de la autopercepción, y un grado de complacencia, tanto en los requerimientos colectivos como individuales.

En referencia con la asociación existente entre la AF y la dimensión AE_hogar, esta evidencia que es posible generar hábitos saludables; en este sentido, los padres deben ser agentes promotores de la AF en sus hijos durante el tiempo extra clase de los estudiantes; de ese modo pueden influir positivamente tanto en su desarrollo emocional como físico. Por consiguiente, han de inducirlos hacia la práctica de la AF, con independencia en lo referente a sus carencias o particularidades, a fin de que mejoren también su AE; estas apreciaciones concuerdan con las expuestas por Castillo, Balaguer y García (2007), en cuanto a que los padres también pueden contribuir en el crecimiento físico y en la mejora de la AE de sus hijos. Además, se pueden aprovechar los espacios en los cuales los integrantes de la familia comparten sus alimentos, su tiempo libre, los juegos de diversión, la televisión, el paseo de fin de semana, el uso de las TIC, entre otras, las cuales preservan algunos valores culturales, pero que no se contraponen a las formas de crianza contemporáneas (O. Herrera, Bedoya y Alviar, 2019).

La presente investigación permitió evidenciar que es posible efectuar diagnósticos oportunos que permitan correlacionar aspectos de la AF y la AE, a fin de diseñar y aplicar actividades específicas a través de la EF, que proporcionen una mejoría de la vida estudiantil, haciéndolas con responsabilidad ética, tanto desde el docente como desde el colegio y el hogar (Jiménez, Martínez, Miró y Sánchez, 2008); al aumentar la frecuencia de ejecución de AF, se generan efectos positivos sobre la AE estudiantil (Biddle \& Asare, 2011). Por consiguiente, el docente de EF ha de promover la AF en sus clases, de modo que los estudiantes incrementen su participación de manera significativa; las actividades se han de orientar a la mejora personal y al fomento de las relaciones interpersonales, de tal forma que promuevan la confianza, el respeto, el aprendizaje cooperativo, la vida saludable, el incremento de la AE y se mengüe la agresividad (Munevar et al., 2019).

En sintesis, el incremento de la AF tiene múltiples beneficios, entre ellos: contribuye con el 
darrollo físico del individuo, incrementa su capacidad motriz, aumenta la AE en sus distintas dimensiones; en particular, incrementa la autoestima general, la cual está también asociada con la autoestima social (Bracho, 2007); y puede generar una considerable disminución de la agresividad, en sus diferentes manifestaciones (Munevar et al., 2019). En consecuencia, es pertinente promover la práctica de AF de los escolares en diversos escenarios; en particular, con el incremento de AF en la clase de educación física, y también desde el hogar.

\section{Conclusiones}

Con fundamento en los hallazgos logrados a partir de la evidencia empírica, se concluye que aproximadamente el $50 \%$ de los participantes presentó bajos niveles de AE, atribuibles a su poca ejecución de AF; en el resto, un incremento de la práctica de AF generó un aumento considerable de su AE; por eso se requiere promoverla desde la escuela y el hogar. En estas circunstancias, se hace urgente diseñar e implementar programas que propicien un aumento significativo de la AF en los escolares adolescentes.

De forma global, en el grupo de estudio se evidenció que existe una correlación positiva, pero moderada, entre la AF y las cuatro dimensiones de AE; de allí se concluye que, en tanto los adolescentes aumenten su AF mejorarán su AE, ya sea que lo hagan en su interacción al interior de la clase de EF o con el apoyo de sus familiares en el hogar o en la comunidad.

Este trabajo ha permitido diagnosticar de manera pertinente el estado de la AE de los participantes, encontrándose que los niveles de AE se pueden mejorar con la formulación y ejecución más frecuente de actividades de tipo físico, tanto en la institución educativa como en el horario extra clase, con el apoyo de los miembros del hogar.

La AF ha de potenciar una educación destinada a generar una vida estudiantil más saludable y ha de proporcionar herramientas útiles para la vida futura del estudiante. Las clases de EF, como parte de tal educación, deben ser proactivas y ofrecer mayores oportunidades para que el adolescente ejecute AF con más frecuencia y, en consecuencia, mejore su AE; asimismo, la EF puede aportar orientaciones tendientes a mejorar las actitudes estudiantiles acerca de la escogencia de una dieta salubre y la realización de AF entre los infantes y los escolares adolescentes. Por consiguiente, se recomienda que la AF sea promocionada tanto en las aulas, en particular dentro de la clase de EF, como en espacios y tiempos extra clase.

Es perentorio que, desde la institución educativa, el hogar y los espacios de convivencia ciudadana, se incremente la ejecución de AF para lograr más altos niveles de AE en los escolares adolescentes y fortalecer su desarrollo físico; además, la AF genera beneficios adicionales asociados con la mengua de la agresividad en sus diferentes manifestaciones, la formación de personas saludables para el entorno social y la promoción de hábitos tendientes a evitar los actos cargados de violencia, las ideaciones suicidas, la agresividad, las bebidas alcohólicas o el consumo de sustancias que mengüen la salud del adolescente.

Para terminar, de este estudio, se pueden desprender algunas otras investigaciones, entre ellas: evaluar la incidencia de aplicar diversos programas destinados a medir el efecto de la AF sobre la mejora de la $\mathrm{AE}$, mediante la aplicación de modelos de regresión lineal, a fin de 
generar evidencia causal del impacto de la AF sobre la AE, o a través de un diseño investigativo cuasi-experimentación; explorar el impacto de realizar AF frecuentemente sobre los niveles de $A E$ en el sector rural y poblaciones vulnerables; investigar sobre la asociación que pueda darse entre la AF, el índice de masa corpórea y la AE sobre escolares de la primera infancia o en otros rangos de edad.

\section{| Referencias}

Akandere, M. y Tekin, A. (2005). Efectos del ejercicio sobre la ansiedad. Publice standard, 478(1), 1-9. Recuperado de http://www.sobreentrenamiento.com/PublicE/Articulo.asp?ida=478

Alcantará, J.A. (2007). Educar la autoestima, métodos, técnicas y actividades. Barcelona, España: CEAC.

Amoedo, N. A. y Juste, M. R. P. (2016). Influencia de la actividad física y el sobrepeso en el rendimiento académico: revisión teórica. Sportis: Revista Técnico-Científica del Deporte Escolar, Educación Física y Psicomotricidad, 2(1), 147-161.

Barrios, H., Peña, L. J. y Cifuentes, R. (septiembre-diciembre, 2019). Emociones y procesos educativos en el aula: una revisión narrativa. Revista Virtual Universidad Católica del Norte, (58), 202-222. https://doi.org/10.35575/rvucn.n58a11

Bernal, I. R. (septiembre, 2006). Influencia de la autoestima en la mejora de la resistencia en adolescentes. La aproximación entre la teoría y la práctica. Revista Internacional de Medicina y Ciencias de la Actividad Física y el Deporte, 6(23), 131-137.

Biddle, S. \& Asare, M. (2011). Physical activity and mental health in children and adolescents: a review of reviews. British journal of sports medicine, 45(11), 886-895. https://doi.org/10.1136/bjsports-2011-090185

Borda, M., Santos, M. A., Martínez, H., Meriño, E., Sánchez, J. y Solano, S. (2016). Percepción de la imagen corporal y su relación con el estado nutricional y emocional en escolares de 10 a 13 años de tres escuelas en Barranquilla (Colombia). Revista Salud Uninorte, 32(3), 472-482.

Bracho, M. V. (2007). Autoestima y motivaciones sociales en estudiantes de educación superior. Venezuela: Universidad Rafael Urdaneta

Brinkmann, H., Segure, T. y Solar, M. (1989). Adaptación, estandarización y elaboración de las normas para el inventario de Coopersmith. Revista Chilena de Psicología, 10(1), 63-71.

Burbano, V. y Valdivieso, M. (2016). Inferencia Estadística Básica, apoyo al estudio independiente. Tunja, Colombia: UPTC.

Campillo, N. C., Zafra, A. O. y Redondo, A. B. (junio, 2008). Relaciones entre la práctica de actividad 
física y el autoconcepto, la ansiedad y la depresión en chicas adolescentes. Cuadernos de psicología del deporte, 8(1), 61-78.

Castillo, I., Balaguer, I. y García, M. (2007). Efecto de la práctica de actividad física y de la participación deportiva sobre el estilo de vida saludable en la adolescencia en función del género. Revista de psicología del deporte, 16(2), 201-210.

Congreso de la República de Colombia. (1982). Ley 23, sobre los Derechos de autor. Recuperado de https://propiedadintelectual.unal.edu.co/fileadmin/recursos/innovacion/docs/normatividad_pi/ley23_1982.pdf

Congreso de la República de Colombia. (2008). Ley 1266, por la cual se dictan las disposiciones generales del Hábeas data. Recuperado de http://www.secretariasenado.gov.co/senado/basedoc/ley_1266_2008.html

CooperSmith, S. (1976). The antecedents of self-esteem. San Francisco, Estados Unidos: Freeman -company.

Delgado, P., Bastian, C., Jerez, D., Cofré, A. y Martínez, C. (enero-junio, 2019). Relación entre sobrepeso, obesidad y niveles de autoestima en escolares. Retos: nuevas tendencias en educación física, deporte y recreación, 35(1), 67-70.

Dishman, R., Felton, G., Hales, D. \& Sward, D. (2006). Physical self- concept and self-esteem mediate cross-sectional relations of physical activity and sport participation with depression symptoms among adolescent girls. Health Psychology, 25(3), 396-407. http://doi.org/10.1037/0278-6133.25.3.396

Echeverri, G. C. (2015). Percepciones de los adolescentes frente a las influencias psicosociales que inciden en sus hábitos alimentarios. Revista Virtual Universidad Católica del Norte, (45), 181-195.

Estévez, E., Martínez, B. y Musitu, G. (septiembre, 2006). La autoestima en adolescentes agresores y víctimas en la escuela: La perspectiva multidimensional. Psichosocial intervención, 15(2), 223-232.

Estévez, M., Muros, J., Torres, B., Pradas, F., Zurita, F. y Cepero, M. (2015). Influencia de la composición corporal y la aceptación por las clases de educación física sobre la autoestima de niños de 14-16 años de Alicante, España. Nutrición Hospitalaria, 31(4), 1519-1524.

Fairclough, S. \& Stratton, G. (2005). Physical education makes you fit and healthy. Physical education's contribution to young people's physical activity levels. Health education research, 20(1), 14-23. https://doi.org/10.1093/her/cyg101

Franco, E., Coterón, J. y Gómez, V. (2017). Promoción de la actividad física en adolescentes: rol de la motivación y autoestima. PSIENCIA. Revista Latinoamericana de Ciencia Psicológica, 9(2), $1-15$. 
Gallego, C. L., Medina, M. T. y Montoya, L. P. M. (2008). Historia de abuso sexual y su relación con depresión, autoestima y consumo de sustancias psicoactivas en estudiantes de media vocacional del municipio de Caldas Antioquia, Colombia. 2007. Revista CES Medicina, 22(1), 17-27.

Guillón, M. S., Crocq, M. A. \& Bailey, P. E. (2007). Nicotine dependence and self-esteem in adolescents with mental disorders. Addictive Behaviors, 32(4), 758-764. https://doi.org/10.1016/j.addbeh.2006.06.018

Hernández, S., Fernández, C. y Baptista, L. (2003). Metodología de la Investigación. Ciudad de México, México: Mc.Graw-Hill.

Herrera, M. F., Álvarez, C., Sánchez, B., Herrera, E., Villalobos, G. y Vargas, J. (2019). Análisis de sobrepeso y obesidad, niveles de actividad física y autoestima en la niñez del II ciclo escolar del cantón central de Heredia, Costa Rica. Población y Salud en Mesoamérica, 17(1), 1-19.

Herrera, O., Bedoya, L. M. y Alviar, M. M. (mayo-agosto, 2019). Crianza contemporánea: formas de acompañamiento, significados y comprensiones desde las realidades familiares. Revista Virtual Universidad Católica del Norte, (57), 40-59. doi: https://doi.org/10.35575/rvucn.n57a4

Jaramillo, L. G., Quilindo, A. M. y Paz, W. F. (2017). Manejo del conflicto en el tiempo-lugar del recreo. Revista Virtual Universidad Católica del Norte, (50), 204-223. Recuperado de http://revistavirtual.ucn.edu.co/index.php/RevistaUCN/article/view/820/1338

Jiménez, M. G., Martínez, P., Miró, E. y Sánchez, A.I. (junio, 2008). Bienestar psicológico y hábitos saludables: ¿están asociados a la práctica de ejercicio físico? International Journal of Clinical and Health Psychology, 8(1), 185-202.

Kowalski, K. C., Crocker, P. R. \& Kowalski, N. P. (1997). Convergent validity of the physical activity questionnaire for adolescents. Pediatric exercise science, 9(4), 342-352.

Lizarazo, L. (2018). Relación entre los niveles de actividad física y la autoestima en estudiantes de grado noveno de la Institución Educativa Gustavo Romero (Trabajo de grado de maestría). Universidad Pedagógica y Tecnológica de Colombia. Tunja, Colombia.

Londoño, L. F., Estrada, E. P. y Alvarado, J. A. (mayo-agosto, 2018). Caracterización de las problemáticas de convivencia de los estudiantes de secundaria de los colegios lasallistas, del distrito área metropolitana Medellín, en los componentes: socio demográfico y de convivencia. Revista Virtual Universidad Católica del Norte, (54), 5-17.

Martínez, D., Martínez, V., Pozo, T., Welk, G., Villagra, A., Calle, M., Marcos, A. y Veiga, O. (mayo-junio, 2009). Fiabilidad y validez del cuestionario de actividad física $P A Q-A$ en adolescentes españoles. Rev. Esp. Salud Pública, 83(3), 427-439.

Mesías, A. A. (2017). Propiedades Psicométricas del Inventario de Autoestima de Coopersmith en 
adolescentes de la provincia de Huallaga. (Trabajo de grado de pregrado). Universidad César vallejo. Trujillo, Perú.

Montoya, A. A. y Pinto, D. A. (agosto, 2016). Nivel de actividad física según el cuestionario PAQ-A En escolares de secundaria en dos colegios de San Martin de Porres-Lima. Revista Herediana de Rehabilitación, 1(1), 21-31.

Moreno, J. A., Cervelló, E. y Moreno, R. (enero, 2008). Importancia de la práctica físico-deportiva y del género en el autoconcepto físico de los 9 a los 23 años. International Journal of Clinical and Health Psychology, 8(1), 171-183.

Mullis, R. L. \& Chapman, P. (2000). Age, gender, and self-esteem differences in adolescent coping style. The Journal of Social Psychology, 140(4), 539-541. https://doi.or$\mathrm{g} / 10.1080 / 00224540009600494$

Munevar, S. K., Burbano, V. M. y Flórez, J. A. (enero-abril, 2019). La actividad física como alternativa de formación para disminuir la agresividad escolar: un estudio comparativo. Revista Virtual Universidad Católica del Norte, (56), 141-160.

Organización Mundial de la Salud -OMS-. (2010). Recomendaciones Mundiales sobre Actividad Física. Suiza: OMS.

Organización Mundial de la Salud -OMS-. (2018). Actividad física Prensa. Recuperado de: http://www.who.int/mediacentre/factsheets/fs385/es/

Organización Mundial de la Salud -OMS-. (s.f.). Desarrollo en la adolescencia. Recuperado de http://www.who.int/maternal_child_adolescent/topics/adolescence/dev/es/

Orth, U., Trzesniewski, K. H. \& Robins, R. W. (2010). Self-Esteem Development From Young Adulthood to Old Age: A Cohort Sequential Longitudinal Study. Journal of Personality and Social Psychology, 98(4), 645-658. http://dx.doi.org/10.1037/a0018769

Pacheco, J., Luarte, C., Pérez, S. y Linz, L. (julio-diciembre, 2015). Incidencia en la autoestima, a partir de Una intervención pedagógica basada en el en el karate tradicional, en estudiantes de una Universidad del Sur de Chile. Revista Ciencias de la Actividad Física, 16(2), 79-85.

Pérez, R. y Smith, V. (2008). Comunicación Intergeneracional: El Cuestionario de Percepción de la Comunicación Intergeneracional (CPCI) en el Contexto Costarricense. Interamerican Journal of Psychology, 42(3), 589-603.

Pérez, R., Aznar, M., Nuviala, R. \& Nuviala, A. (2019). Evaluation of extracurricular sports activities as an educational element for sustainable development in educational institutions. Sustainability, 11(12), 3474.

Piñeros, M. y Pardo, C. (diciembre, 2010). Actividad física en adolescentes de cinco Ciudades colombianas: resultados de la Encuesta Mundial de Salud a Escolares. Revista de Salud 
Pública, 12(6), 903-914.

Pope, A.W., McHale, S. \& Craighead, W.E. (1988). Self-Esteen Enhancement With children and adolescents. New York, Estados Unidos: Pergamon Press.

Prewitt, J. (1984). A croos cultural study of the reliability of the coopersmith self steem Inventory. Educational and Phychological Measurement, 44(3), 575-581.

Redondo, J., Luzardo, M., Larrotta, R. y Rangel, K. (2015). Diferencias en comportamientos agresivos entre adolescentes colombianos. Revista Virtual Universidad Católica del Norte, (44), 5-14. Recuperado de http://revistavirtual.ucn.edu.co/index.php/RevistaUCN/article/view/612/1147

Rodríguez, C. y Caño, A. (2012). Autoestima en la adolescencia: análisis y estrategias de Intervención. Internal J. Psychology and Psychological Therapy, 12(3), 389-403.

Salas, M., Levette, M., Redondo, J. y Luzardo, M. (2017). Ideación suicida en adolescentes víctimas de cyberbullying del instituto empresarial Gabriela Mistral de Floridablanca, Santander. Revista Virtual Universidad Católica del Norte, (51), 80-97. Recuperado de http://revistavirtual.ucn.edu.co/index.php/RevistaUCN/article/view/844/1362

Schmalz, D., Deane, G. \& Birch, L. (december, 2007). A Longitudinal Assessment of the Links Between Physical Activity and Self-Esteem in Early Adolescent Non-Hispanic Females. Journal of Adolescent Health, 41(6), 559-565. https://doi.org/10.1016/j.jadohealth.2007.07.001

Schneider, M., Fridlund, G. \& Cooper, D. (january, 2008). Physical activity and physical Self -concept among sedentary adolescent females: An intervention study. Psychology of sport and exercise, 9(1), 1-14. https://doi.org/10.1016/j.psychsport.2007.01.003

Vásquez, A. A., Zelaya, C. y García, J. (2019). Análisis de sobrepeso y obesidad, niveles de actividad física y autoestima en escolares de San Pedro Sula, Honduras. MHSalud, 16(2), 58-71.

Vásquez, J. E. y López, E. (septiembre-diciembre, 2018). El perdón como reconstructor de subjetividades y contextos. Revista Virtual Universidad Católica del Norte, (55), 67 -85.

Vidarte, J. A., Vélez, C., Sandoval, C. y Mora, M. L. (enero-junio, 2011). Actividad física: estrategia de promoción de la salud. Revista Hacia la Promoción de la Salud, 16(1), 202-218.

Zurita, F., Castro, M. Rodríguez, S., Cofré, C., Chacón, B. R., Martínez, A. y Muros, J. J. (2017). Actividad física, obesidad y autoestima en escolares chilenos: Análisis mediante ecuaciones estructurales. Revista médica de Chile, 145(3), 299-308. 